\title{
Acute bronchopulmonary infection due to Streptococcus milleri in a child with cystic fibrosis
}

\author{
A Cade, M Denton, K G Brownlee, N Todd, S P Conway
}

\begin{abstract}
An 8 year old girl with cystic fibrosis had severe respiratory disease associated with chronic Pseudomonas aeruginosa bronchopulmonary infection. Despite regular courses of intravenous antipseudomonal antibiotics, she continued to deteriorate over 18 months with persistent productive cough, worsening respiratory function, and increasing oxygen dependence. During her 11th admission Streptococcus milleri was isolated from sputum cultures in addition to $P$ aeruginosa. She failed to respond to antipseudomonal antibiotics but improved dramatically with the addition of intravenous benzylpenicillin. A1though $S$ milleri is considered a normal mouth commensal and its isolation from sputum of cystic fibrosis patients is of uncertain significance, it was associated with clinically significant infection in this child. $S$ milleri was eradicated with antibiotic treatment and clinical improvement has been maintained.

(Arch Dis Child 1999;80:278-279)
\end{abstract}

Cystic Fibrosis Unit,
St James's University

Hospital, Beckett

Street, Leeds LS7 9TF,

UK

A Cade

K G Brownlee

S P Conway

Microbiology

Department, St

James's University

Hospital

N Todd

Microbiology

Department, Leeds

General Infirmary,

Leeds, UK

M Denton

Correspondence to: Dr Cade.

Accepted 6 August 1998

Keywords: cystic fibrosis; Streptococcus milleri; Pseudomonas aeruginosa

An 8 year old girl had been diagnosed at 2 years of age with cystic fibrosis (homozygous for $\Delta \mathrm{F} 508)$ following persistent cough, failure to thrive, and a positive sweat test (sodium $84 \mathrm{mmol} / 1$, chloride $98 \mathrm{mmol} / 1$, sweat volume $241 \mathrm{mg}$ ). Pseudomonas aeruginosa was first isolated at 3 years of age and 12 months later she was started on three monthly elective courses of intravenous antibiotics for chronic $P$ aeruginosa bronchopulmonary infection. Between April 1996 and October 1997 she required 10 admissions to hospital for acute on chronic respiratory exacerbations. On each occasion she had received at least two weeks of intravenous antipseudomonal antibiotics. Regular sputum cultures consistently grew both mucoid and non-mucoid strains of $P$ aerugi-

Table 1 Respiratory function tests

\begin{tabular}{lllll}
\hline Function & April 1996 & September 1997 & October 1997 & fanuary 1998 \\
\hline PEFR (1/min) & 68 & 90 & 190 & 150 \\
VC (litres) (\%) & $0.70(53)$ & $0.48(32)$ & $1.08(71)$ & $0.99(63)$ \\
FVC (litres) (\%) & $0.65(49)$ & $0.55(37)$ & $1.22(80)$ & $1.04(66)$ \\
FEV $_{1}$ (litres) (\%) & $0.37(30)$ & $0.33(24)$ & $0.73(51)$ & $0.65(44)$ \\
FEV $_{1} /$ FVC\% & $56(-37)$ & $61(-33)$ & $60(-34)$ & $62(-31)$ \\
FEF $_{25-75}$ (litres) (\%) & $0.13(8)$ & $0.13(7)$ & $0.26(14)$ & $0.25(13)$
\end{tabular}

$S$ milleri was isolated and treated in September 1997.

$\star$ Percentage of expected.

PEFR, peak expiratory flow rate; VC, vital capacity; FVC, forced vital capacity; $\mathrm{FEV}_{1}$, forced expiratory volume in one second; $\mathrm{FEF}_{25-75}$, forced expiratory flow between $25 \%$ and $75 \%$ of vital capacity. nosa. On two occasions she also received intravenous flucloxacillin because Staphylococcus aureus had been isolated from sputum. Between admissions she had eight courses of oral ciprofloxacin. Maintenance treatment included aerosolised colomycin and oral flucloxacillin. Despite this intensive approach, she remained chronically symptomatic with persistent productive cough, offensive halitosis, and significantly decreased exercise tolerance. Assessment for lung transplantation was considered as her forced expiratory volume in one second $\left(\mathrm{FEV}_{1}\right)$ was consistently around $30 \%$ of predicted. In October 1997 she was admitted to hospital requiring supplemental oxygen treatment. She had a fever with a raised white cell count $\left(18.6 \times 10^{9} / 1\right.$, neutrophils 14.4$)$ and a $\mathrm{C}$ reactive protein (CRP) of $112 \mathrm{mg} / \mathrm{l}$. The fever did not abate and the CRP remained high despite five days of intravenous colomycin and ceftazidime. Sputum cultures taken at admission grew Streptococcus milleri as well as $P$ aeruginosa. Intravenous benzylpenicillin ( $180 \mathrm{mg} / \mathrm{kg} /$ day) and metronidazole $(22.5 \mathrm{mg} / \mathrm{kg} /$ day $)$ were started in addition to antipseudomonal antibiotics. Over the next two weeks her temperature, white cell count, and CRP returned to normal and she showed a dramatic improvement in symptoms, respiratory function (table 1 ), and chest radiography appearance. She received a further six week course of oral penicillin V (40 mg/kg/day) on discharge (October 1997).

Since this event she has returned to hospital only once for her regular intravenous antipseudomonal antibiotics and has remained well. She has not grown $S$ milleri in her sputum since treatment, the offensive halitosis has resolved completely, and her $\mathrm{FEV}_{1}$ has almost doubled.

\section{Discussion}

$S$ milleri (also classified as $S$ consellatus, $S$ intermedius, and $S$ anginosus) is a facultative anaerobic $\alpha$ haemolytic streptococcus found as a normal mouth commensal. It is clinically associated with abscess formation, in particular dental, lung, brain, and intra-abdominal, often mixed with other organisms. A recent report on the cause of pulmonary infections highlighted the frequency of isolation of $S$ milleri in pneumonia, lung abscess, and empyema, but not in patients with cystic fibrosis. ${ }^{1} S$ milleri is very rarely identified from cough swabs or sputum of patients with cystic fibrosis because it is not specifically looked for. In fact infection with $S$ milleri has never been described before in cystic fibrosis patients with the exception of an isolated case report of a 21 year old man with bilateral brain abscesses caused by $S$ intermedius. In that 
case, sputum culture did not grow the organism. ${ }^{2}$ The fact that we isolated $S$ milleri from the sputum of our patient was a chance finding. If present on culture it tends to form minute colonies that are generally indistinguishable from the many other normal mouth commensals, and is usually overlooked.

There was a clear association between the introduction of benzylpenicillin and the dramatic clinical response for our patient. Metronidazole had been started at the same time because of the possibility of lung abscess formation and the recognised association between $S$ milleri and the presence of anaerobes. Unfortunately sputum cultures were not routinely analysed for anaerobes, so their presence was never confirmed in this case.

Many antipseudomonal antibiotics have very poor activity against $S$ milleri and their regular use may have selected in its favour. Similarly, the activity of flucloxacillin against these organisms is uncertain. Imipenem does have activity against $S$ milleri and anaerobes but our patient had not received this agent for six months because of the emergence of $P$ aerugi- nosa strains resistant to carbopenems. Although $S$ miller $i$ is the least likely of the viridans group of streptococci to develop resistance to the more commonly used antibiotics in hospital practice, its resistance has been reported.

$S$ milleri is rarely isolated from the sputum of cystic fibrosis patients. If it is, its significance may be underestimated and must be considered in the context of the patient's clinical condition. Our experience suggests that it can be a true pathogen and its eradication can result in significant clinical and radiological improvement. For cystic fibrosis patients that do not respond to antipseudomonal antibiotic treatment, microbiological measures to look actively for anaerobes, in particular $S$ milleri may be warranted.

1 Shinzato T, Saito A. The Streptococcus milleri group as a cause of pulmonary infections. Clin Infect Dis 1995;21:S23843.

2 Duffner PK, Cohen E. Cystic fibrosis with brain abscess. Arch Neurol 1979;36:27-8.

3 Doern GV, Ferraro MJ, Brueggemann AB, Ruoff KL. Doern GV, Ferraro MJ, Brueggemann AB, Ruoff KL.
Emergence of high rates of antimicrobial resistance among Emergence of high rates of antimicrobial resistance among
viridans group streptococci in the United States. Antimicrob Agents Chemother 1996;40:891-4. 